\title{
Orientações legais para diagnóstico da mastofauna em Estudos de Impactos Ambientais ajudam? Análise crítica no caso do Estado de São Paulo, Brasil
}

\section{Ana Paula Rodrigues Pinto', Maristela Camolesi², Eduardo Roberto Alexandrino $^{3}$}

1Pós-Graduação latu sensu em Gerenciamento Ambiental. Escola Superior de Agricultura “Luiz de Queiroz”. Universidade de São Paulo. Campus Piracicaba. ITAITI Consultoria Ambiental.

${ }^{2}$ Graduação em Licenciatura em Ciências Biológicas. Universidade Federal de São Carlos. Campus Sorocaba.

${ }^{3}$ Programa de Pós-Graduação Interunidades em Ecologia Aplicada. Laboratório de Ecologia, Manejo e Conservação da Fauna Silvestre. Departamento de Ciências Florestais. Escola Superior de Agricultura “Luiz de Queiroz”. Universidade de São Paulo. Campus Piracicaba. E-mail: eduardoalexandrino@hotmail.com

Resumo. A apresentação de Estudo de Impacto Ambiental (EIA) é obrigatória em processos de licenciamento ambiental de diversos empreendimentos e para atividades consideradas potencialmente causadoras de significativa degradação ao meio ambiente. O diagnóstico ambiental do local a sofrer intervenção é uma das atividades técnicas que devem ser apresentadas em um EIA e considera, dentre outros parâmetros ambientais, o levantamento da mastofauna. A Companhia Ambiental do Estado de São Paulo (CETESB) é quem norteia tais levantamentos neste estado. O presente estudo revisou o diagnóstico da mastofauna de 20 EIAs iniciados em 2013 e 2014, atentando-se para possíveis deficiências no levantamento e análise de dados. Os resultados observados foram comparados com as diretrizes impostas em documentos legais da CETESB válidos a partir de 2014, para verificar o quanto estes documentos poderiam ser suficientes para minimizar tais deficiências em EIAs futuros. As principais deficiências encontradas foram a (1) não realização de levantamentos em campo, (2) listas de espécies incompletas, (3) não declaração sobre a forma e local de registro das espécies em campo, (4) ausência de caracterização da assembleia de mamíferos amostrada, (5) não declaração do esforço e suficiência amostral realizado, (6) ausência de análises quantitativas, bem como (7) ausência de comparação dos resultados obtidos com áreas similares. Argumenta-se que as quatro primeiras deficiências poderão ser evitadas em trabalhos futuros com os documentos legais vigentes na CETESB. Porém, as três restantes permanecerão, pois não há orientações robustas e claras nestes documentos sobre tais temas. Logo, salienta-se a necessidade de adequações nestes documentos e que o corpo técnico da CETESB continue atento nas avaliações dos diagnósticos realizados em EIAs no estado.

Palavras-chave: Consultoria ambiental; Decisão da Diretoria CETESB; Paisagens antrópicas; Gestão ambiental; Mamíferos.
Recebido:

$11 / 09 / 2017$

Aceito:

$19 / 11 / 2017$

Publicado:

31/12/2017

Acesso aberto

Artigo completo

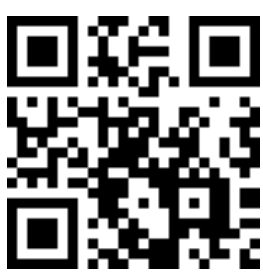

ORCID

(1) 0000-0002-2812-2099

Ana Paula Rodrigues

Pinto

(D) 0000-0001-9466-5975

Maristela Camolesi

(D) $0000-0003-3088-4524$

Eduardo Roberto

Alexandrino 
Abstract. Does legal guidelines for mammals diagnosis in Environmental Impact Assessment help? Critical review in the case of São Paulo State, Brazil. The Environmental Assessment (EA) is a mandatory document that every enterprise interested to install and operate in Brazil should submit to the government to get environmental licensing. All localities where the enterprises are intended to produce impacting changes must be surveyed, producing the environmental diagnosis for the EA. Mammals is one of the environmental variables that should be considered. In São Paulo State, the Environmental Company of the State of São Paulo (CETESB) guides all environmental surveys. We performed a mammal diagnosis review of 20 EAs, starting from 2013-2014, and we searched for possible shortcoming on data sampling and analysis. Then, the results were compared with CETESB's legal rules, valid since 2014, to verify whether their own documents would minimize the occurrence of those observed shortcomings on future EAs. The main shortcomings observed were (1) absence of field sampling, (2) incomplete species list, (3) non-specification of each species record type and location of each species on field, (4) lack of assemblage characterization, (5) non-specification of field sampling effort and sampling sufficiency, (6) lack of quantitative analysis, (7) lack of assemblage comparisons with similar locations. The first four shortcomings should be avoided in future EA with the current CETESB legal rules. However, the last three shortcomings should stay occurring, because there are no robust and clear orientations about those subjects on CETESB rules. Thus, we highlight that adjustments should occur on CETESB rules and their professionals should stay alert in future assessments of mammals diagnosis performed in EAs in the state.

Keywords: Environmental consulting; Environmental management; Human-modified landscape; Decision making process; Mammals.

\section{Introdução}

O processo de Avaliação de Impacto Ambiental (AIA) é uma ferramenta que visa a minimizar impactos oriundos de diversas atividades antrópicas (Glasson e Salvador, 2000; Dias e Sánchez, 2001; Sánchez, 2006; Sánchez e Croal, 2012). Foi introduzido no Brasil por meio da Lei $\mathrm{n}^{\circ}$ 6.938/1981 (Brasil, 1981), mas foi em 1986 que passou a ser aplicado de forma efetiva por meio de requisitos estabelecidos pela Resolução CONAMA nº 001/1986 (Brasil, 1986). Atualmente qualquer empreendimento a ser instalado no Brasil que cause impacto ambiental significativo deve passar pelo processo AIA (Dias e Sánchez, 2001; Sánchez e Croal, 2012).

Segundo Sánchez (2006), a atividade central da AIA é a elaboração do
Estudo de Impacto Ambiental (EIA), um estudo prévio de caráter multidisciplinar que fornece bases para a análise da viabilidade ambiental do empreendimento (Dias e Sánchez, 2001). De acordo com a Resolução CONAMA nº 001/1986, dentre as atividades técnicas que devem compor um EIA há o diagnóstico ambiental da área de influência do empreendimento planejado (Brasil, 1986, ver art. $6^{\circ}$ ). O diagnóstico é a caracterização da situação ambiental antes da implantação do empreendimento (Sánchez, 2006; Almeida et al., 2015) e considera fatores do meio físico, biótico e socioeconômico (Brasil, 1986). A elaboração do diagnóstico deve ser minuciosa, uma vez que ele fornecerá informações básicas necessárias à identificação, previsão e avaliação dos impactos ambientais, bem como a definição 
dos programas de gestão ambiental do empreendimento (i.e., medidas mitigadoras, compensatórias e programas de monitoramento) (Glasson e Salvador, 2000; Sánchez, 2006; Almeida et al., 2015). O diagnóstico ainda servirá como um banco de dados para futuras comparações, caso o empreendimento seja implantado (e.g., Straube et al., 2010), o que eleva sua importância.

No caso do meio biótico, seu diagnóstico envolve especificamente a caracterização da fauna e flora do local sob influência do empreendimento, demandando, então, a atuação de diversos especialistas (i.e., botânicos, ornitólogos, mastozoólogos, herpetólogos). Embora nos últimos anos houve apelo por uma melhor qualidade dos cursos superiores brasileiros voltados a qualificar tais profissionais (graduações, pós-graduações lato sensu e strictu sensu nas áreas de Biologia e Ecologia, e.g., Martins et al., 2007; Pardini et al., 2013), ainda são comuns diagnósticos da fauna mal conduzidos, com esforços amostrais insuficientes e com o emprego de métodos de coleta de dados e análises inadequados (Vasconcelos, 2006; Mazzolli et al., 2008; Silveira et al., 2010; Scherer, 2011; Almeida et al., 2015; Almeida et al., 2016; Alexandrino et al., 2016; Almeida et al., 2017). Na tentativa de evitar tais problemas, ao longo dos anos os órgãos ambientais brasileiros passaram a instituir, por meio de normativas, procedimentos metodológicos e analíticos básicos aos diagnósticos da fauna em EIAs. Por exemplo, no âmbito federal há a Instrução Normativa IBAMA $n^{\circ}$ 146/2007 (Brasil, 2007, ver arts. $4^{\circ}$ e $5^{\circ}$ ). No caso dos órgãos estaduais que possuem competência técnica, há a possibilidade de serem instituídos documentos legais similares, válidos unicamente em seu território.

No Estado de São Paulo, por muitos anos, o próprio corpo técnico do Departamento Estadual de Proteção dos Recursos Naturais (DEPRN) e do Departamento de Avaliação de Impacto Ambiental (DAIA), ambos extintos em maio de 2009 (São Paulo, 2009) e integrantes da Secretaria de Estado do Meio Ambiente, é quem elaborava as diretrizes mínimas que orientava os consultores na condução de laudos de fauna em estudos com finalidades de licenciamento ambiental. Por estas diretrizes serem lançadas particularmente a cada estudo, por meio dos Termos de Referências, o processo de licenciamento dos empreendimentos muitas vezes era demorado. Para ajudar, entre 2000 e 2015, a Portaria DEPRN no 42/2000 (São Paulo, 2000) surgiu com o intuito de informar procedimentos básicos que deveriam ser realizados em laudos de fauna de todo tipo de estudo ambiental. No entanto, tal portaria possuía caráter generalista e era considerado pouco instrutivo. Após a nova atribuição da Companhia Ambiental do Estado de São Paulo - CETESB (São Paulo, 2009), como o principal órgão responsável pela análise destes estudos e concessão das licenças ambientais no estado, seu corpo técnico passou a publicar normativas, com validade legal, sobre os procedimentos mínimos a serem considerados nos EIAs. Destacam-se a Decisão de Diretoria CETESB n ${ }^{\circ}$ 217/2014/I (São Paulo, 2014) e a Decisão de Diretoria CETESB $n^{\circ}$ 167/2015/C (São Paulo, 2015), aqui referidas, respectivamente, como DD 217/2014/I e DD 167/2015/C. A proposta destas DDs era a de evitar a realização de diagnósticos insuficientes, o que compromete a boa execução dos EIAs e, consequentemente, contribui com atrasos nos processos de licenciamento.

Dentre os grupos da fauna que devem ser diagnosticados nos EIAs, consta os mamíferos (São Paulo, 2014; São Paulo, 2015). A fragmentação e perda de habitat dos biomas brasileiros põem em risco diversas espécies (Chiarello et al., 2008; Magioli et al., 2015, 2016), além da pressão da caça ilegal a que estão sujeitos (Cullen et al., 2001; Pardini et al., 2003; Chiarello et al., 2008; Palmeira e Trinca, 2012). Logo, dado o grau de ameaça e a importância ecológica deste grupo, instruções que visem a uma coleta e análise de dados eficaz, no tempo disponível para a condução dos EIAs, tornam-se altamente necessárias. Embora o objetivo das instruções normativas seja aumentar a qualidade dos diagnósticos da fauna, estes documentos 
legais só atingirão seus objetivos se avaliações forem realizadas ao longo do tempo de sua vigência, identificando os pontos pendentes de melhorias (e.g., Alexandrino et al., 2016).

Neste contexto, a fim de colaborar no aprimoramento técnico-científico das diretrizes mínimas voltadas à elaboração de diagnósticos de mastofauna de EIAs no Estado de São Paulo, o presente estudo primeiramente elaborou uma análise crítica elencando possíveis deficiências cometidas, que colocam em risco a qualidade do diagnóstico. Em seguida, os resultados obtidos foram comparados com as diretrizes mínimas propostas pela DD 217/2014/I e DD 167/2015/C, com o intuito de verificar o quanto estes documentos, em sua atual redação, poderiam ser suficientes para minimizar tais deficiências nos próximos diagnósticos a serem realizados no estado. Por fim discutem-se ainda alguns pontos críticos dessas DDs.

\section{Material e métodos}

Foram selecionados EIAs que estavam disponíveis para download no site da CETESB (CETESB, 2016), entre os dias 9 e 31 de janeiro de 2016, data em que se procedeu a busca pelos estudos. Uma vez que são feitos constantes avanços científicos na área de inventário e análise de mastofauna (e.g., Srbek-Araujo e Chiarello, 2013; Lyra-Jorge et al., 2014; Magioli et al., 2014a; Marques et al., 2014), optou-se pela seleção somente dos estudos que possuíam data de abertura de processo no órgão ambiental nos anos de 2013 e 2014, a fim de minimizar a seleção de estudos com disparidade no expertise de seus consultores devido à diferença dos anos de sua realização. Desse modo, foram selecionados 20 EIAs (Tabela 1).

Os inventários de mastofauna presentes no item "Diagnóstico Ambiental do Meio Biótico" de cada EIA foram analisados seguindo um roteiro de perguntas com respostas fechadas (Tabela 2). Este roteiro orientou as análises sobre a qualidade da fonte de dados considerados no diagnóstico, procedimentos de levantamento de dados em campo e forma de análise de dados, três objetos do estudo que determinam o sucesso do diagnóstico e a avaliação da fauna estudada (e.g., Silveira et al., 2010; Straube et al., 2010; Scherer, 2011). Estes objetos são tratados no item "Fauna Terrestre", do anexo único, da DD 217/2014/I (São Paulo, 2014), e no art. 3º, da DD 167/2015/C (São Paulo, 2015). Em seguida, buscou-se identificar se os resultados obtidos estariam de acordo com os procedimentos propostos nas DDs.

Por se tratar de EIAs, foram considerados apenas os procedimentos propostos do "Nível 1" de complexidade da Fauna Terrestre da DD 217/2014/I, do qual traz as recomendações consideradas mais robustas. Salienta-se também que foram desconsideradas das análises informações sobre as áreas de influência específicas onde os dados de mastofauna foram coletados em cada EIA (i.e., Área de Influência Indireta - AII, Área de Influência Direta - AID, Área Diretamente Afetada ADA), sendo que o que interessou foi responder as questões propostas na Tabela 2 , independente do local da fonte dos dados (exceto para perguntas 7 e 10). 
Tabela 1. Relação dos Estudos de Impacto Ambiental (EIAs) analisados.

\begin{tabular}{|c|c|c|c|c|c|c|}
\hline $\begin{array}{c}\mathbf{N}^{\mathbf{o}} \\
\text { Processo }\end{array}$ & Data & $\begin{array}{l}\text { Empresa de } \\
\text { consultoria } \\
\text { Ambiental } \\
\end{array}$ & Empreendedor & Empreendimento & $\begin{array}{c}\text { Tipologia do } \\
\text { empreendimento }\end{array}$ & Município \\
\hline 03_2013 & Dez/2012 & $\begin{array}{l}\text { SAVI Assessoria, } \\
\text { Consultoria em } \\
\text { Mineração e Meio } \\
\text { Ambiente Ltda. }\end{array}$ & $\begin{array}{c}\text { Guapiara } \\
\text { Mineração } \\
\text { Indústria e } \\
\text { Comércio Ltda. }\end{array}$ & $\begin{array}{c}\text { Extração de } \\
\text { calcário da Mina } \\
\text { Coqueiro }\end{array}$ & $\begin{array}{l}\text { Extração de } \\
\text { minério }\end{array}$ & Guapiara \\
\hline 15_2013 & Set/2013 & $\begin{array}{c}\text { CPEA - } \\
\text { Consultoria, } \\
\text { Planejamento e } \\
\text { Estudos } \\
\text { Ambientais Ltda. }\end{array}$ & $\begin{array}{c}\text { EcoUrbis } \\
\text { Ambiental S.A. }\end{array}$ & $\begin{array}{l}\text { Ampliação da } \\
\text { Central de } \\
\text { Tratamento de } \\
\text { Resíduos Leste }\end{array}$ & Aterro sanitário & São Paulo \\
\hline 30_2013 & Fev/2014 & $\begin{array}{c}\text { RESITEC } \\
\text { Serviços } \\
\text { Industriais Ltda. }\end{array}$ & $\begin{array}{c}\text { TCL - Tecnologia } \\
\text { e Construções } \\
\text { Ltda. }\end{array}$ & \begin{tabular}{|c|} 
Central de \\
tratamento e \\
destinação de \\
resíduos sólidos \\
domiciliares em \\
regime de \\
codisposição com \\
resíduos \\
industriais classe \\
IIA e IIB e \\
Autoclave - \\
\end{tabular} & Aterro sanitário & Amparo \\
\hline 80_2013 & Set/2013 & $\begin{array}{c}\text { JGP Consultoria e } \\
\text { Participações } \\
\text { Ltda. }\end{array}$ & \begin{tabular}{|} 
Secretaria de \\
Transportes - \\
Prefeitura \\
Municipal de São \\
José dos Campos
\end{tabular} & $\begin{array}{l}\text { Projeto da Via } \\
\text { Cambuí }\end{array}$ & Estrada & $\begin{array}{l}\text { São José } \\
\text { dos } \\
\text { Campos }\end{array}$ \\
\hline 81_2013 & $*$ & $\begin{array}{c}\text { ConAm } \\
\text { Consultoria } \\
\text { Ambiental Ltda. }\end{array}$ & \begin{tabular}{|} 
Jardim Fazenda \\
Cantagalo - \\
Empreendimentos \\
Imobiliários SPE \\
Ltda.
\end{tabular} & \begin{tabular}{|c|} 
Loteamento \\
Residencial e \\
Comercial Jardim \\
Fazenda \\
Cantagalo \\
\end{tabular} & $\begin{array}{l}\text { Projeto } \\
\text { urbanístico }\end{array}$ & $\begin{array}{l}\text { Ribeirão } \\
\text { Preto }\end{array}$ \\
\hline 87_2013 & 2014 & $\begin{array}{l}\text { P.A. Brasil - } \\
\text { Consultoria e } \\
\text { Planejamento } \\
\text { Ambiental S/C } \\
\text { Ltda. }\end{array}$ & $\begin{array}{c}\text { Paiva Ramos } \\
\text { Empreendimentos } \\
\text { Imobiliários Ltda. }\end{array}$ & \begin{tabular}{|c} 
Projeto \\
urbanístico \\
Fazenda Paiva \\
Santos
\end{tabular} & $\begin{array}{l}\text { Projeto } \\
\text { urbanístico }\end{array}$ & Osasco \\
\hline 88_2013 & Nov/2013 & \begin{tabular}{|l} 
Walm Engenharia \\
e Tecnologia \\
Ambiental Ltda.
\end{tabular} & \begin{tabular}{|c|} 
Logística \\
Ambiental de São \\
Paulo S/A - \\
LOGA
\end{tabular} & $\begin{array}{l}\text { Estação de } \\
\text { transbordo } \\
\text { Anhanguera }\end{array}$ & Aterro sanitário & São Paulo \\
\hline 89_2013 & $\mathrm{Abr} / 2015$ & $\begin{array}{l}\text { Walm Engenharia } \\
\text { e Tecnologia } \\
\text { Ambiental Ltda. }\end{array}$ & \begin{tabular}{|c|} 
Empresa \\
Metropolitana de \\
Transportes \\
Urbanos de São \\
Paulo S/A - \\
EMTU
\end{tabular} & \begin{tabular}{|} 
Sistema Integrado \\
Metropolitano de \\
Veículo Leve \\
sobre Trilhos \\
(Fase 2) Trecho \\
Conselho Nébias \\
- Valongo
\end{tabular} & Estrada & Santos \\
\hline
\end{tabular}


Tabela 1. Continuação.

\begin{tabular}{|c|c|c|c|c|c|c|}
\hline $\begin{array}{c}\mathbf{N}^{\circ} \\
\text { Processo }\end{array}$ & Data & $\begin{array}{l}\text { Empresa de } \\
\text { consultoria } \\
\text { Ambiental } \\
\end{array}$ & Empreendedor & Empreendimento & $\begin{array}{c}\text { Tipologia do } \\
\text { empreendimento }\end{array}$ & Município \\
\hline 124_2013 & I & \begin{tabular}{|c|} 
PLANSERVI \\
Engenharia, \\
Companhia \\
Brasileira de \\
Projetos e \\
Empreendimentos \\
- COBRAPE
\end{tabular} & $\begin{array}{c}\text { Prefeitura } \\
\text { Municipal de São } \\
\text { José dos Campos }\end{array}$ & $\begin{array}{c}\text { Sistema viário Via } \\
\text { Banhado }\end{array}$ & Estrada & $\begin{array}{l}\text { São José } \\
\text { dos } \\
\text { Campos }\end{array}$ \\
\hline 182_2013 & Fev/2014 & $\begin{array}{l}\text { MKR Tecnologia, } \\
\text { Serviços, } \\
\text { Indústria e } \\
\text { Comércio Ltda. }\end{array}$ & $\begin{array}{c}\text { Logum Logística } \\
\text { S.A. }\end{array}$ & Dutovia de etanol & Duto & $\begin{array}{c}29 \\
\text { municípios } \\
\text { do Est. de } \\
\text { São Paulo } \\
\end{array}$ \\
\hline 189_2013 & Abr/2015 & \begin{tabular}{|} 
HIDROSTUDIO \\
Engenharia, \\
THEMAG \\
Engenharia e \\
Gerenciamento \\
Ltda.
\end{tabular} & $\begin{array}{l}\text { Departamento de } \\
\text { Águas e Energia } \\
\text { Elétrica - DAEE }\end{array}$ & $\begin{array}{c}\text { Barragens para } \\
\text { disponibilidade } \\
\text { hídrica na Bacia } \\
\text { do Rio Jaguari }\end{array}$ & $\begin{array}{c}\text { Obra hidráulica } \\
\text { para exploração } \\
\text { de recursos } \\
\text { hídricos }\end{array}$ & $\begin{array}{l}\text { Pedreira } \\
\text { Campinas } \\
\text { Amparo }\end{array}$ \\
\hline 194_2013 & Nov/2014 & $\begin{array}{l}\text { Ziguia Engenharia } \\
\text { Ltda. }\end{array}$ & $\begin{array}{c}\text { Piracicaba } \\
\text { Ambiental S.A. }\end{array}$ & \begin{tabular}{|c|} 
Aterro sanitário - \\
Central de \\
Tratamento de \\
Resíduos \\
Palmeiras \\
\end{tabular} & Aterro sanitário & Piracicaba \\
\hline 258_2013 & Set/2014 & \begin{tabular}{|} 
MKR Tecnologia, \\
Serviços, \\
Indústria e \\
Comércio Ltda.
\end{tabular} & $\begin{array}{c}\text { Canárias } \\
\text { Administradora } \\
\text { de Bens Ltda. }\end{array}$ & $\begin{array}{c}\text { Loteamento Misto } \\
\text { Canárias }\end{array}$ & $\begin{array}{l}\text { Projeto } \\
\text { urbanístico }\end{array}$ & Porto Feliz \\
\hline 416_2013 & Dez/2014 & $\begin{array}{l}\text { Multiambiente } \\
\text { Serviços Ltda. }\end{array}$ & $\begin{array}{l}\text { Mineradora } \\
\text { Jundu Ltda. }\end{array}$ & $\begin{array}{l}\text { Ampliação da } \\
\text { área de lavra da } \\
\text { Mina Olho } \\
\text { D'Água }\end{array}$ & $\begin{array}{l}\text { Extração de } \\
\text { minério }\end{array}$ & $\begin{array}{c}\text { Bom } \\
\text { Sucesso de } \\
\text { Itararé }\end{array}$ \\
\hline 53_2014 & Mar/2015 & $\begin{array}{c}\text { RESITEC } \\
\text { Serviços } \\
\text { Industriais Ltda. }\end{array}$ & $\begin{array}{c}\text { TCL - } \\
\text { Tecnologia e } \\
\text { Construções Ltda. }\end{array}$ & \begin{tabular}{|c|} 
Central de \\
tratamento e \\
destinação de \\
resíduos sólidos \\
domiciliares em \\
regime de \\
codisposição com \\
resíduos \\
industriais Classe \\
I, IIA e IIB e \\
autoclave - \\
\end{tabular} & Aterro sanitário & Adamantina \\
\hline 113_201 & Fev/2015 & $\begin{array}{c}\text { PRIME } \\
\text { Engenharia e } \\
\text { Comércio Ltda. }\end{array}$ & $\begin{array}{c}\text { Companhia de } \\
\text { Saneamento } \\
\text { Básico do Estado } \\
\text { de São Paulo - } \\
\text { SABESP }\end{array}$ & \begin{tabular}{|} 
Interligação entre \\
as Represas \\
Jaguari (Bacia do \\
Paraíba do Sul) e \\
Atibainha (Bacias \\
PCJ)
\end{tabular} & \begin{tabular}{|} 
Obra hidráulica \\
para exploração \\
de recursos \\
hídricos
\end{tabular} & $\begin{array}{c}\text { Santa Isabel } \\
\text { Igaratá } \\
\text { Nazaré } \\
\text { Paulista }\end{array}$ \\
\hline
\end{tabular}


Tabela 1. Continuação.

\begin{tabular}{|c|c|c|c|c|c|c|}
\hline $\begin{array}{c}\mathbf{N}^{\mathbf{o}} \\
\text { Processo }\end{array}$ & Data & $\begin{array}{l}\text { Empresa de } \\
\text { consultoria } \\
\text { Ambiental } \\
\end{array}$ & Empreendedor & Empreendimento & $\begin{array}{c}\text { Tipologia do } \\
\text { empreendimento }\end{array}$ & Município \\
\hline 115_2014 & $*$ & $\begin{array}{c}\text { GEOTEC } \\
\text { Consultoria } \\
\text { Ambiental Ltda. }\end{array}$ & $\begin{array}{c}\text { Concessionária } \\
\text { Rota das } \\
\text { Bandeiras }\end{array}$ & \begin{tabular}{|c|} 
Perimetral de \\
Itatiba \\
(SPI081/360) - \\
segmento \\
rodoviário entre o \\
cruzamento das \\
Rod. Romildo \\
Prado (SP-063) e \\
Eng. Constâncio \\
Cintra (SP-360) \\
até a Rod. D. \\
Pedro I (SP-065)
\end{tabular} & Estrada & Itatiba \\
\hline 160_2014L & Dez/2014 & $\begin{array}{l}\text { MKR Tecnologia, } \\
\text { Serviços, } \\
\text { Indústria e } \\
\text { Comércio Ltda. }\end{array}$ & Grupo Pacaembu & $\begin{array}{l}\text { Loteamento } \\
\text { Jardim Cristo } \\
\text { Redentor }\end{array}$ & $\begin{array}{c}\text { Projeto } \\
\text { urbanístico }\end{array}$ & $\begin{array}{l}\text { Ribeirão } \\
\text { Preto }\end{array}$ \\
\hline 238_2014[ & Dez/2015 & $\begin{array}{c}\text { TN Ambiental } \\
\text { Engenharia } \\
\text { Química S/S Ltda. }\end{array}$ & $\begin{array}{c}\text { Ipiranga } \\
\text { Agroindustrial } \\
\text { S.A. }\end{array}$ & $\begin{array}{c}\text { Ampliação do } \\
\text { parque industrial e } \\
\text { processo } \\
\text { produtivo }\end{array}$ & $\begin{array}{c}\text { Complexo e } \\
\text { unidades } \\
\text { industriais e } \\
\text { agroindustriais }\end{array}$ & Мососа \\
\hline 345_2014]J & Jun/2015 & \begin{tabular}{|c|} 
Dfreire \\
Planejamento e \\
Consultoria Ltda.
\end{tabular} & \begin{tabular}{|c|} 
Lote 5 \\
Desenvolvimento \\
Urbano S.A. \\
\end{tabular} & \begin{tabular}{|c|} 
Empreendimento \\
Urbanístico \\
Campo Verde
\end{tabular} & $\begin{array}{c}\text { Projeto } \\
\text { urbanístico }\end{array}$ & Jarinu \\
\hline
\end{tabular}

Processo CETESB: número do processo de licenciamento ambiental para consulta na Companhia Ambiental do Estado de São Paulo (CETESB); Data: mês e ano em que o EIA foi realizado; Empresa de consultoria ambiental: razão social da empresa de consultoria ambiental responsável pelo EIA; Empreendedor: razão social do empreendedor; Empreendimento: denominação do empreendimento; Tipologia do empreendimento: estabelecida com base no art. $2^{\circ}$, da Resolução CONAMA n ${ }^{\circ} 001 / 1986$; Município: cidade do Estado de São Paulo em que o empreendimento está localizado. * mês e ano não informados no EIA. No entanto, pelo número do Processo CETESB sabe-se que os estudos ocorreram entre 2013 e 2015.

Tabela 2. Roteiro utilizado para a análise crítica dos Estudos de Impactos Ambientais (EIAs) selecionados.

\section{Identificação do EIA:}

Número do processo CETESB; Data em que o estudo de mastofauna foi realizado; Nome da empresa de consultoria ambiental responsável pelo estudo; Nome do empreendedor; Denominação do empreendimento; Tipologia do empreendimento com base no art. $2^{\circ}$, da Resolução CONAMA $n^{\circ}$ 001/1986; Município do Estado de São Paulo em que o empreendimento está localizado.

\begin{tabular}{|c|c|c|}
\hline \multicolumn{2}{|c|}{ Perguntas que pautaram a análise } & \multirow[t]{2}{*}{ Respostas possíveis } \\
\hline & Fonte de dados e coleta de dados em campo & \\
\hline 1 & O estudo considerou dados de mamíferos em seu diagnóstico? & sim/não \\
\hline 2 & O estudo utilizou dados secundários? & sim/não \\
\hline 3 & O estudo realizou coleta de dados em campo? & sim/não \\
\hline 4 & $\begin{array}{l}\text { O estudo realizou campanha de levantamento de campo no } \\
\text { período seco e no período chuvoso? }\end{array}$ & $\begin{array}{l}\text { só no seco/só no chuvoso/em } \\
\text { ambos }\end{array}$ \\
\hline
\end{tabular}


Tabela 2. Continuação.

\begin{tabular}{|c|c|c|}
\hline \multicolumn{2}{|c|}{ Perguntas que pautaram a análise } & \multirow{2}{*}{$\begin{array}{c}\text { Respostas possíveis } \\
\operatorname{sim} / \text { não }\end{array}$} \\
\hline 5 & $\begin{array}{l}\text { O estudo declarou as metodologias utilizadas para as coletas de } \\
\text { campo? }\end{array}$ & \\
\hline 6 & O estudo declarou o esforço amostral empregado em campo? & sim/não \\
\hline 7 & O estudo apresenta a curva de acúmulo de espécies?* & sim/não \\
\hline 8 & $\begin{array}{l}\text { O estudo apresenta qual foi a forma de registro de cada espécie } \\
\text { em campo (registro direto e/ou indireto)? }\end{array}$ & sim/não \\
\hline \multirow[t]{2}{*}{9} & O estudo declarou o local de registro de cada espécie em campo? & sim/não \\
\hline & Forma de análise e apresentação dos dados de campo & \\
\hline 10 & O estudo declarou a riqueza registrada em campo?** & sim/não \\
\hline 11 & $\begin{array}{l}\text { O estudo apresentou lista de espécie contendo família, nome } \\
\text { científico e nome popular das espécies registradas? }\end{array}$ & sim/não \\
\hline 12 & $\begin{array}{l}\text { O estudo realizou uma descrição, em parâmetros ecológicos e/ou } \\
\text { funcionais, das espécies registradas em campo? }\end{array}$ & sim/não \\
\hline 13 & $\begin{array}{l}\text { O estudo apresenta e comenta sobre as espécies ameaçadas de } \\
\text { extinção registradas em campo? }\end{array}$ & sim/não \\
\hline 14 & $\begin{array}{l}\text { O estudo apresenta e comenta sobre as espécies exóticas } \\
\text { registradas em campo? }\end{array}$ & sim/não \\
\hline 15 & $\begin{array}{l}\text { O estudo apresenta e comenta sobre as espécies endêmicas } \\
\text { registradas em campo? }\end{array}$ & sim/não \\
\hline 16 & $\begin{array}{l}\text { O estudo adota alguma análise quantitativa sobre a assembleia de } \\
\text { mamíferos amostrada em campo (i.e, frequência de ocorrência, } \\
\text { abundância relativa ou índice de diversidade)? }\end{array}$ & sim/não \\
\hline 17 & $\begin{array}{l}\text { O estudo comparou a mastofauna registrada em campo com a de } \\
\text { outras áreas semelhantes? }\end{array}$ & sim/não \\
\hline 18 & $\begin{array}{l}\text { O estudo analisou a sensibilidade das espécies registradas em } \\
\text { campo? }\end{array}$ & sim/não \\
\hline 19 & $\begin{array}{l}\text { O estudo analisou o estado de conservação ambiental da área } \\
\text { estudada tomando os mamíferos como bioindicadores? }\end{array}$ & sim/não \\
\hline
\end{tabular}

* para cada EIA que usou curvas, também atentou-se se qual o número máximo de espécies observadas que constavam nas curvas apresentadas, e se foi usado estimadores de riqueza (dados apenas da ADA e AID).

** para cada EIA analisado também foi anotado o número de espécies registradas em campo na ADA e AID, locais onde geralmente são feitas amostragens em campo. Este dado foi utilizado para compreender os motivos do uso das demais formas de análises de dados.

\section{Resultados}

Considerando as tipologias de empreendimento sugeridas no art. $2^{\circ}$, da Resolução CONAMA n ${ }^{001 / 1986}$ (Brasil, 1986), os EIAs analisados fazem parte de sete tipologias diferentes, tendo sido variável o número de estudos por tipologia: aterro sanitário $(\mathrm{n}=5)$, projeto urbanístico $(n=5)$, estradas $(n=4)$, obra hidráulica para exploração de recursos hídricos $(\mathrm{n}=2)$, extração de minério $(\mathrm{n}=2)$, duto
( $\mathrm{n}=1)$ e complexo e unidades industriais e 
agroindustriais $(\mathrm{n}=1)$. Apesar de serem 20 EIAs, apenas 17 tiveram sua área de influência limitada a um único município (e.g., destaca-se um EIA onde a totalidade ou parte de 29 municípios do estado fez parte a sua área de influência, ver EIA $n^{\circ}$ 182_2013 na Tabela 1). Assim, numa análise espacial, percebe-se que a maior parte dos locais sob influência dos empreendimentos analisados estão inseridos na extensão original da Mata Atlântica e a menor parte na extensão do Cerrado (Figura 1).

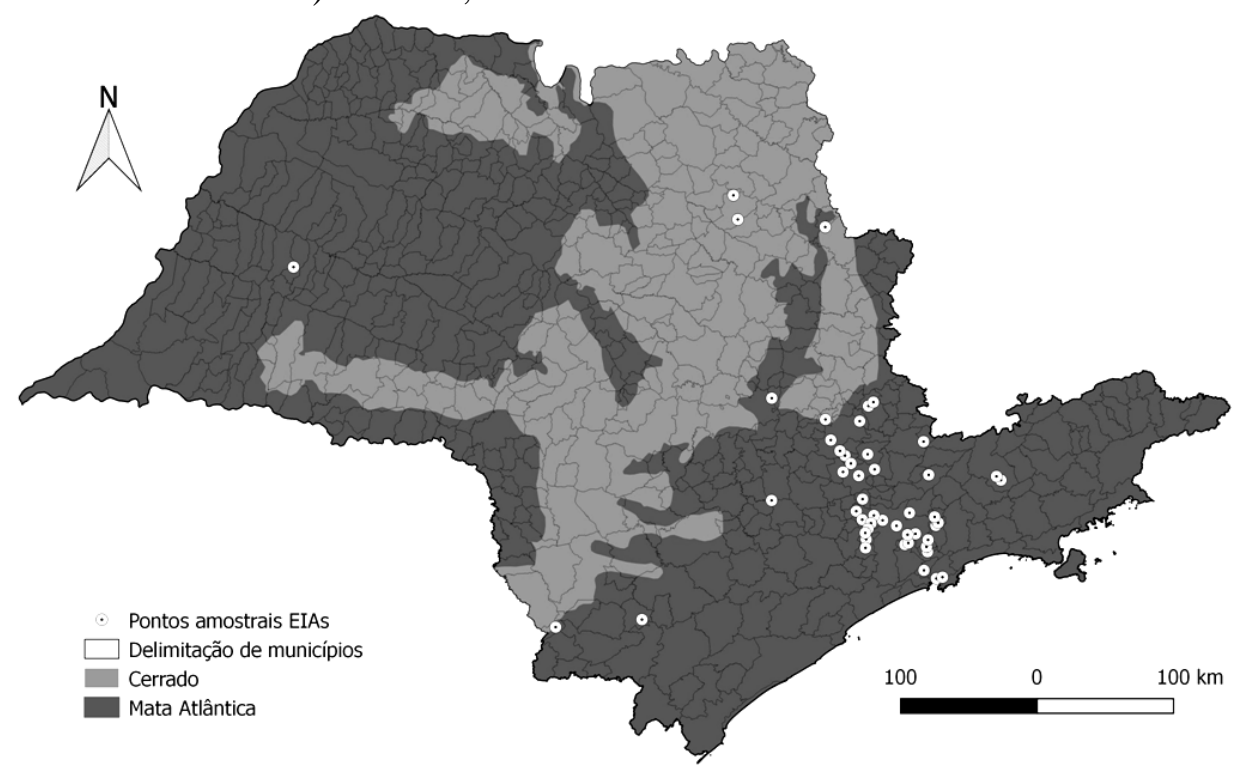

Figura 1. Localização nos municípios e em relação à extensão original dos biomas existentes no Estado de São Paulo onde os Estudos de Impactos Ambientais dos 20 empreendimentos analisados previam intervenções ambientais. Mapa base disponível em MMA (2017).

\section{Deficiências na fonte de dados e coleta de dados em campo}

Todos os 20 EIAs analisados consideraram dados de mamíferos em seu diagnóstico. Sobre a fonte dos dados, embora todos tenham utilizado dados obtidos de referências bibliográficas (i.e., dados secundários), apenas 17 EIAs (85\%) realizaram suas próprias coletas de dados em campo (i.e., dados primários, seja coletado na ADA, AID ou AII).

Considerando apenas os EIAs que realizaram coletas em campo, observou-se que apenas $41,1 \%$ deles (total de 7 estudos) realizaram coletas no período seco (abril a setembro) e chuvoso (outubro a março), 23,5\% (4) dos estudos realizaram apenas no período seco e $29,4 \%$ (5) apenas no chuvoso. Embora todos os estudos declararam as metodologias utilizadas para as coletas em campo, dois estudos não declararam o local de registro onde cada espécie foi observada (e.g., se observada em fragmentos florestais, pastagens, canaviais, etc), e três estudos não forneceram detalhes sobre a forma de registro de cada espécie em campo (i.e., observação direta, pegadas, armadilha fotográfica, etc.). O número médio de espécies registradas com as amostragens em campo, considerando ADA e AID dos estudos, foi de 15,5 espécies (máximo 33, mínimo 9).

Em 76,4\% (13 estudos) dos estudos houve o uso da curva de acúmulo de espécie para representar o incremento de espécies amostradas em relação ao esforço amostral. No entanto, três destes estudos deixaram de declarar o esforço amostral empregado nas coletas em campo, sendo que esta informação não fica clara apenas por meio das curvas usadas. O número de espécies considerada na elaboração das curvas foi variável, sendo que houve um 
EIA que apresentou curva com apenas duas espécies até EIAs que apresentaram curvas que possuíam mais de 20 espécies (ver Tabela 3 em documentos suplementares).

\section{Deficiências na análise e apresentação dos dados de campo}

Todos os 17 estudos que realizaram levantamento de campo declararam a riqueza de espécies registradas. No entanto, o uso de análises mais aprofundadas sobre a assembleia de mamíferos amostrada, bem como o fornecimento de detalhes em cada análise, foi variável entre os estudos. Três EIAs não caracterizaram as espécies com parâmetros ecológicos e/ou funcionais, por exemplo, habitat preferencial de ocorrência, hábitos alimentares, grau de sensibilidade a distúrbios antrópicos, etc. Apenas 58,8\% destes estudos (10) declararam algum tipo de grau de sensibilidade às espécies registradas. Contudo, ressalta-se que em todos os casos esse grau de sensibilidade foi elaborado levando em consideração apenas o expertise do consultor, não tendo sido informada alguma referência bibliográfica de base. Embora todos os estudos explicaram sobre a ocorrência ou ausência de espécies consideradas em alguma categoria de ameaça, apenas 35,3\% (6) fizeram uma mesma análise considerando as espécies endêmicas encontradas em campo. Do mesmo modo, $11,7 \%$ dos estudos (2) não fizeram menção sobre a ocorrência ou ausência de espécies exóticas em campo.

Em relação a análises quantitativas, ao todo, $52,9 \%$ (8) dos EIAs realizaram alguma análise quantitativa. Destes, sete estudos exploraram ao menos a frequência de ocorrência das espécies registradas em campo, ou em relação às unidades amostrais ou em relação aos dias de trabalho de campo realizado. Somente cinco realizaram levantamento da abundância relativa e apenas um aplicou índice de diversidade.

Somente três EIAs realizaram uma comparação da assembleia amostrada em campo com as existentes em demais áreas com características ambientais similares, provenientes de outros trabalhos já realizados. Finalmente, todos os 17 estudos discutiram sobre o grau de conservação das áreas de influência do empreendimento tendo como base o resultado obtido da mastofauna.

\section{Discussões}

Deficiências e a atuação das DDs Fonte de dados e coleta em campo.

Embora tenha sido pouco frequente entre os EIAs analisados, a realização de diagnósticos com base unicamente em dados secundários é uma deficiência grave. A utilidade de dados advindos de referências bibliográficas varia em função da metodologia empregada em cada coleta, das quais muitas vezes não são adequadas para um diagnóstico ambiental num processo de licenciamento ambiental. Por exemplo, tais dados podem ter sido coletados num momento do passado que não mais refletem a assembleia de fauna que ocorre na data em que o EIA está sendo executado. Em paisagens antrópicas, onde a composição, configuração, bem como a substituição, do uso do solo podem influenciar a ocorrência da mastofauna (e.g., Umetsu et al., 2008; Lyra-Jorge et al., 2010), a coleta de dados primários nas áreas de influência do empreendimento será a aproximação mais atual da assembleia ocorrente no período de execução do EIA. Considerando o conteúdo das DDs este tipo de deficiência deverá ser sanado, uma vez que ambos os documentos recomendam que os dados secundários devem ser tratados apenas como complementação dos dados primários (São Paulo, 2014; São Paulo, 2015).

Informar sobre o local onde cada espécie foi registrada em campo é um dado que permite a exploração de demais informações ecológicas sobre a assembleia amostrada (Pardini et al., 2003), tais como, o tipo de habitat em que cada espécie é capaz de ocorrer (e.g., borda ou interior de remanescentes florestais, capoeiras, próximo a corpos d'água), bem como interpretar o nível de tolerância das espécies a usos antrópicos (e.g., áreas de cultivo agrícola, estrada, áreas urbanas etc.). Esse tipo de informação contribuirá diretamente nas etapas seguintes previstas 
no EIA, onde serão feitas a avaliação dos impactos previstos sobre a fauna amostrada, propostas de medidas mitigadoras $\mathrm{e}$ compensatórias e definição de programas de gestão ambiental (Sánchez, 2006). Do mesmo modo, fornecer detalhes sobre a forma como cada espécie foi registrada em campo (e.g., observação direta, vocalização, pegadas, fezes, carcaças, tocas, armadilha, armadilha fotográfica, entrevistas) (e.g., Lyra-Jorge et al., 2014; Magioli et al., 2014b) auxilia na avaliação, por parte do órgão ambiental, se o método de amostragem adotado no habitat em questão foi adequado e reflete nos resultados apresentados (ver Bovendorp et al., 2017), bem como entender se houve bom uso do esforço realizado na coleta em campo (e.g., Silveira et al., 2010; Straube et al., 2010).

Considerando que em alguns EIAs analisados não foram fornecidas as informações supracitadas, e sabendo da importância delas para o diagnóstico e avaliação do estudo por parte da CETESB, espera-se que todas estas informações constem em EIAs futuros, uma vez que todas estas são recomendadas nas DDs vigentes.

Análise de dados. Nos EIAs, um dos propósitos mais comuns em apresentar a riqueza de espécies de cada grupo da fauna registrada em campo é demonstrar a capacidade do local sob análise em comportar a biodiversidade (e.g., Silveira et al., 2010; Straube et al., 2010; Lyra-Jorge et al., 2014; Magioli et al., 2014b). No entanto, Alexandrino et al. (2017) demonstraram que em pequenos remanescentes florestais localizados em paisagem antrópica, a riqueza pode não ser o melhor parâmetro para auxiliar na avaliação do habitat por meio da fauna, deixando de ser um indicador ecológico. Dessa forma, em um diagnóstico ambiental, a caracterização da assembleia da fauna que foi amostrada favorece uma análise mais detalhada e robusta sobre os locais estudados. O primeiro passo para se realizar tal análise é elaborar a listagem de espécies. A partir dela é possível apontar quais são as espécies endêmicas, ameaçadas e exóticas, bem como classificar as espécies, por exemplo, sobre seu hábito alimentar, habitat preferencial de ocorrência, ou demais parâmetros (e.g., massa, tamanho, etc.) que possam ser úteis na identificação da função ecológica de cada espécie (Pardini et al., 2003; Straube et al., 2010; Alexandrino et al., 2017). Mesmo que este tipo de informação não conste no EIA, o fornecimento da lista de espécies, organizada em níveis taxonômicos e com o nome científico e popular, permite que qualquer outro profissional proceda tais análises, auxiliando na avaliação do diagnostico realizado. Ressalta-se que uma das propostas do processo AIA é a participação da sociedade, que tendo acesso ao EIA depois de pronto, poderá apontar falhas e sugerir melhorias no estudo (e.g., Scherer, 2011; Alexandrino et al., 2016), contribuindo com a melhoria da avaliação do local a ser impactado (ver Sánchez, 2006)

Considerando todos os EIAs analisados, foi observado que 77 espécies ameaçadas foram registradas nas áreas de influências dos empreendimentos, o que evidencia o quanto estudos de fauna em EIAs podem contribuir para 0 conhecimento da ocorrência destas espécies (e.g., Azevedo, 2006; Mazzolli et al., 2008). Este resultado também reforça a importância do desenvolvimento de programas de monitoramento da fauna nas etapas seguintes do processo de licenciamento ambiental, a fim de auxiliar na proposição de medidas mitigadoras e na definição de áreas prioritárias para conservação da biodiversidade (e.g., Silveira et al. 2010; Straube et al., 2010). Dessa forma, os diagnósticos devem fazer menção sobre a ocorrência ou ausência de espécies ameaçadas. Além disso, uma vez que vários remanescentes de Cerrado e a Mata Atlântica no estado de São Paulo encontram-se ameaçados pelos avanços antrópicos (e.g., Verdade et al., 2012), as espécies endêmicas destes biomas também têm grande importância na avaliação dos locais. No entanto, como observado nos resultados, muitas vezes não são feitos quaisquer comentários a respeito destas espécies nos EIAs. Do mesmo modo, embora alguns empreendimentos venham 
se instalar em locais já degradados, onde não ocorram mais espécies endêmicas ou ameaçadas, a indicação da ocorrência de espécies exóticas e domésticas em áreas silvestres é um substituto útil para a avaliação do local. Estas espécies competem diretamente ou indiretamente por recursos (i.e., alimento, espaço, etc) com as espécies nativas (e.g., Galetti e Sazima, 2006; Campos et al., 2007), podem elevar a taxa de predação de tais populações (e.g., Alexandrino et al., 2012; Loss et al., 2013), bem como podem prejudicar práticas agrícolas e a regeneração natural de remanescentes florestais (Hegel e Marini, 2013).

Muitos EIAs analisados realizaram de forma superficial a análises da assembleia de mastofauna. No entanto, as DDs vigentes trazem trechos recomendando a caracterização da fauna amostrada em campo. Desse modo, espera-se que análises mais detalhadas, como as supracitadas, passem a ser realizadas em todos os EIAs futuros.

\section{Pontos críticos das DDs}

Curva de acúmulo de espécies da mastofauna. Ambas as DDs solicitam que sejam apresentadas no EIA as curvas de acúmulos de espécies de todas as assembleias da fauna amostrada em campo, uma recomendação que replica uma norma nacional (ver Brasil, 2007). Esta é uma forma visual de representar o incremento de espécies novas ao longo da realização das amostras para avaliar o esforço realizado (Santos, 2003; Magurran, 2004). No entanto, pesquisadores têm levantado discussões sobre a eficiência do uso de curvas de acúmulos de espécies para tal propósito (e.g., Schilling et al., 2012), um tema que extrapola os muros das universidades (e.g., Vasconcelos, 2006; Straube et al., 2010). Por exemplo, em consultorias ambientais pode haver casos em que a área amostrada naturalmente não possui um número elevado de mamíferos (e.g., áreas altamente perturbadas, áreas de pastagens, áreas urbanas), além de que o curto tempo disponível para as amostragens em campo evita uma detecção maior a prováveis espécies ocorrentes (Santos,
2003). Assim, nestes casos seria sensato imaginar que o uso das curvas não acrescentaria muita informação ao diagnóstico do local, se ao invés disso uma boa descrição da comunidade observada e do esforço realizado fosse oferecida. Embora essa fosse uma premissa inicial do presente estudo, foi observado que o uso da curva esteve presente mesmo em EIAs onde o número de espécies relatado foi extremamente baixo, e houve casos onde mesmo tendo sido observada uma quantidade maior de mamíferos o consultor não utilizou tal análise. Isso demonstra que parece não haver um padrão entre os consultores pela escolha ou abstenção do uso das curvas. Além disso, atentando-se aos estudos que usaram curvas, constatouse que $77 \%$ deles (10 EIAs) utilizaram também estimadores de riquezas. Sabendo que estes estimadores sempre irão indicar que o número de espécies esperado é maior que o número de espécies observado (Magurran, 2004), questiona-se porque os consultores estariam fazendo tais análises se em seus relatórios eles declaram que suas áreas amostrais estão inseridas em áreas degradadas e que o tempo disponível em campo foi insuficiente. Estes resultados sugerem que muitos consultores estejam usando tais curvas apenas como um mero cumprimento da legislação.

Neste contexto, levanta-se a dúvida se a requisição pelas curvas de acúmulo de espécies num documento legal estaria de fato auxiliando os mastozoólogos no processo de avaliação ambiental. Logo, recomenda-se que o corpo técnico da CETESB mantenha extrema atenção a cada caso e busque compreender se o uso das curvas é ou não necessário de acordo com a situação ambiental da área amostrada em cada EIA.

\section{Análises mais aprofundadas}

Algumas solicitações das DDs para que haja coletas e análise de dados de campo mais aprofundados ainda necessitam de maiores detalhamentos. Isso leva a crer que estas normativas sozinhas não irão garantir nos EIAs futuros a presença de algumas análises solicitadas. 
Em relação à sazonalidade, sabe-se que nas zonas tropicais importantes processos ecológicos são afetados pela estação do ano, tais como fenologia e frutificação de espécies vegetais, o que influencia na distribuição da mastofauna de um dado local (e.g., Paschoal e Galetti, 1995; Alvez-Costa et al., 2004). Do mesmo modo, sabe-se que os médios e grandes mamíferos ocorrentes em paisagens antropizadas dependem de recursos que estão distribuídos na paisagem (e.g., Magioli et al., 2014a). Dessa forma, quando o objetivo é conhecer a assembleia de mamíferos ocorrentes em tais paisagens, é sensata a recomendação da DD 217/2014/I sobre a realização de amostras na estação seca e chuvosa. No entanto, em áreas antrópicas altamente degradadas no estado de São Paulo (e.g., áreas urbanas e culturas agrícolas), onde o empobrecimento da comunidade de mamíferos pode ser elevada (e.g., Gheler-Costa et al., 2002; Lyra-Jorge et al., 2008) é questionável se a realização de amostras em ambas estações é demasiadamente exagerada. Desse modo, a DD 167/2015/C fixou que apenas nos casos em que o empreendimento prevê a supressão de vegetação nativa em área acima de 10,01 hectares será necessário levantamentos em ambas as estações, e abaixo disso a amostragem poderá ser realizada na estação considerada mais propícia ao encontro da mastofauna no dado local. Porém, mesmo com as DDs vigentes, o corpo técnico da CETESB deverá ter extrema cautela em analisar cada plano de trabalho de cada EIA e lançar recomendações específicas por meio do Termo de Referência.

O uso de parâmetros quantitativos sobre a assembleia amostrada em campo pode auxiliar na interpretação dos dados qualitativos observados (Pardini et al., 2003; Magioli et al., 2014b, Alexandrino et al., 2017). Apesar das DDs requisitarem análises da fauna que necessitem diretamente e indiretamente de dados quantitativos (e.g., diversidade, abundância, interpretação da distribuição espacial das espécies em campo, relação espécie-habitat, relação espécie-sítio amostral, sazonalidade, etc), o texto das DDs não explicitam que tais análises só serão alcançadas por meio de delineamento amostral que permita a coleta de parâmetros quantitativos. É sensato pensar que esta recomendação deva estar implícita na DD, uma vez que se parte do princípio que os profissionais executores dos diagnósticos possuem expertise suficiente. No entanto, de acordo com os resultados aqui obtidos, nem sempre os profissionais utilizam medidas quantitativas. Dessa forma, entende-se que a o corpo técnico da CETESB também deverá atentar-se a ocorrência desta deficiência, e quando reconhecida a necessidade, solicitar, por meio do Termo de Referência, a execução de trabalhos de campo que permitam a coleta de dados quantitativos.

Embora a possibilidade de se estabelecer comparações entre as assembleias amostradas em campo com dados secundários sejam dependentes da natureza das coletas realizadas, observa-se que algumas análises solicitadas ao longo da DD 217/2014/I podem ser alcançadas se tais comparações forem realizadas. Por exemplo, a indicação da ocorrência de espécies não esperadas na área de influência do empreendimento poderá ser alcançada tomando a ocorrência dos mamíferos em levantamentos secundários realizados na região. Esta base de análise pode ser útil também na interpretação dos possíveis corredores ecológicos na região, atentando aos pontos de registro das espécies. Assim como já citado no parágrafo anterior, parte-se do princípio que profissionais possuem conhecimento suficientes para explorar tais dados secundários. No entanto, os poucos estudos que realizaram comparações entre a mastofauna observada no EIA e demais dados regionais, deram mais atenção ao número de espécie observada em cada estudo, limitando o poder da avaliação do EIA. Dessa forma, percebe-se que o corpo técnico da CETESB ainda terá que recomendar tais metodologias de análises aos EIAs, por meio do Termo de Referência.

O "grau de sensibilidade a distúrbios antrópicos” é o parâmetro que mais se aproxima da ideia de indicador 
ecológico, classicamente desejado por ecólogos e tomadores de decisões (Dale e Beyeler, 2001; Niemi e MacDonald, 2004; Alexandrino et al., 2016). Embora seu uso seja recomendado pelas DDs, ainda é incerta a melhor forma de determinar grau de sensibilidade a todas as espécies de mamíferos terrestres. A ideia básica de um parâmetro deste tipo é lançar uma nota que represente o potencial da população de uma dada espécie em sofrer alterações perceptíveis (e.g., pode ser em número de indivíduos, na distribuição espacial, em parâmetros que representem qualidade de saúde nos indivíduos, etc.) seja de forma positiva ou negativa, conforme ocorram distúrbios antrópicos no local sob análise (Dale e Beyeler, 2001). Os resultados aqui obtidos mostraram que os EIAs que usaram tal classificação lançaram níveis de sensibilidade de forma subjetiva, o que pode resultar em discordâncias entre consultores e os analistas ambientais da CETESB em relação a interpretação da qualidade ambiental do local sob análise (e.g., Alexandrino et al., 2016). Percebe-se que atualmente as DDs não informam o suficiente sobre este parâmetro, o que levanta questões sobre como realizá-lo. Assim, caso este parâmetro continue a ser solicitado no diagnóstico de fauna, recomenda-se que o corpo técnico da CETESB solicite, por meio do Termo de Referência, que cada EIA estabeleça uma metodologia de classificação das espécies em níveis de sensibilidade. Esta classificação pode ser feitas com base em fatores ecológicos coletados em campo (i.e., dados sobre a distribuição espacial, dados quantitativos), bem como ter base em informações sobre as características funcionais da espécie (e.g., guilda trófica, habitat preferencial de ocorrência, etc.), seu status de conservação em escala estadual ou nacional e informações sobre endemismo (e.g., Alexandrino et al., 2017; Teresa e Casati, 2017). Tais critérios precisaram ser bem justificados e detalhados permitindo que a CETESB avalie a forma com que os níveis foram estabelecidos.

\section{Considerações finais}

A análise ambiental sobre os locais amostrados com base na fauna observada é algo que sempre foi solicitado nos diagnósticos. O poder de argumentação sobre tal qualidade é variável em função da qualidade dos dados coletados em campo. No entanto, o expertise do consultor também influencia nas análises realizadas. Como o tempo disponível para estes estudos geralmente são poucas semanas, sabe-se que a facilidade na execução de um diagnóstico mais detalhado está ligada ao tempo de atuação deste profissional e aproximação dele com as inovações científicas. Este é um problema que ocorre em EIAs realizados em várias partes do mundo (e.g., Wegner et al., 2005; Khera e Kumar, 2010). Logo, considerando a variação no aprofundamento de cada análise realizada nos EIAs, entende-se que não é possível esperar que instruções normativas forneçam todos os requisitos possíveis aos executores dos EIAs, o que faz com que os analistas ambientais continuem atentos em suas avaliações, e igualmente preparados para identificar erros analíticos que vão além da recomendação das DDs. O art. 6 ${ }^{\circ}$, da DD 167/2015/C, do qual permite que os técnicos responsáveis pela avaliação do EIA solicitem complementações, deverá amparar situações onde estes profissionais não percebam robustez nas avaliações ambientais realizadas por meio da mastofauna.

Os resultados obtidos neste artigo demonstram que mesmo num estado brasileiro onde é observada uma organização institucional elevada dentro do órgão que lida com os processos de Avaliação de Impactos Ambientais (CETESB, 2016), é possível observar que a publicação de regulamentações para análise de fauna ainda está longe de obter excelência, demandando revisões constantes em seu texto. 


\section{Agradecimentos}

Este estudo é oriundo da monografia de conclusão de Curso de Especialização em Gerenciamento Ambiental (ESALQ/USP) da primeira autora, logo, agradecemos os ricos comentários dos membros da banca examinadora que permitiram o avanço na qualidade do presente documento. Agradecemos ao Laboratório de Métodos Quantitativos, do Departamento de Ciências Florestais da ESALQ/USP, pelo uso dos computadores e espaço físico e a Jefferson Lordelo Polizel pelo apoio na elaboração da figura.

\section{Referências}

Alexandrino, E. R.; Luz, D. T. A.; Maggiorini, E. V.; Ferraz, K. M. P. M. B. Nest stolen: the first observation of nest predation by an invasive exotic marmoset (Callithrix penicillata) in an agricultural mosaic. Biota Neotropica, v. 12, n. 2, p. 211-215, 2012. https://dx.doi.org/10.1590/S1676-0603201200 0200021

Alexandrino, E. R.; Buechley, E. R.; Piratelli, A. J.; Ferraz, K. M. P. M B.; Moral, R. A.; Şekercioğlu, Ç. H.; Silva, W. R.; Couto, H. T. Z. Bird sensitivity to disturbance as an indicator of forest patch conditions: an issue in environmental assessments. Ecological Indicators, v. 66, p. 369-381, 2016. https://dx.doi.org/10.1016/j.ecolind.2016.02.006

Alexandrino, E. R.; Buechley, E. R.; Karr, J. R.; Ferraz, K. M. P. M.. B.; Ferraz, S. F. B.; Couto, H. T. Z.; Şekercioğlu, Ç. H. Bird based Index of Biotic Integrity: assessing the ecological condition of Atlantic Forest patches in humanmodified landscape. Ecological Indicators, v. 73, p. 662-675, $2017 . \quad$ https://dx.doi.org/ 10.1016/j.ecolind.2016.10.023

Almeida, A. N.; Sertão, A. C.; Soares, P. R. C.; Angelo, H. Deficiências no diagnóstico ambiental dos estudos de impacto ambiental (EIA). Revista de Gestão Ambiental e Sustentabilidade, v. 4, n. 1, p. 33-48, 2015. https://dx.doi.org/10.5585/geas.v4i2.168

Almeida, A. N.; Oliveira, N. B.; Silva, J. C. G. L.; Angelo, H. Principais deficiências dos Estudos de Impacto Ambiental. Revista Brasileira de Gestão Ambiental e
Sustentabilidade, v. 3, n. 4, p. 3-14, 2016. https://dx.doi.org/10.21438/rbgas.030401

Almeida, A. N.; Kanieski, M. R.; Soares, P. R. C.; Angelo, H. Principais problemas na previsão e avaliação de impactos ambientais nos Estudos de Impacto Ambiental (EIAs): uma aplicação da análise de correlação canônica. Revista Brasileira de Gestão Ambiental e Sustentabilidade, v. 4, n. 7, p. 31-42, 2017. https://dx.doi.org/10.21438/rbgas.040704

Alvez-Costa, C. P.; Fonseca, G. A. B.; Christófaro, C. Variation in the diet of the brown-nosed coati (Nasua nasua) in Southeastern Brazil. Journal of Mammalogy, $\begin{array}{llll}\text { v. } 85, & \text { n. } 3, & \text { p. } 478-482, & 2004 .\end{array}$ https://dx.doi.org/10.1644/1383945

Azevedo, M. A. G. Contribuição de estudos para licenciamento ambiental ao conhecimento da avifauna de Santa Catarina, Sul do Brasil. Biotemas, v. 19, n. 1, p. 93-106, 2006. Disponível em: <https://periodicos.ufsc.br/ index.php/biotemas/article/view/21341/19247>. Acesso em: 20 abr. 2017.

Bovendorp, R. S.; McCleery, R. A.; Galetti, M. Optimising sampling methods for small mammal communities in Neotropical rainforests. Mammal Review, v. 47, n. 2, p. 148-158, 2017. https://dx.doi.org/10.1111/ mam.12088

Brasil. Lei no 6.938, de 31 de agosto de 1981 . Dispõe sobre a Política Nacional do Meio Ambiente, seus fins e mecanismos de formulação e aplicação, e dá outras providências. Disponível em: <http://www.planalto.gov.br/ ccivil_03/leis/L6938.htm>. Acesso em: 20 abr. 2017.

Brasil. Resolução CONAMA no 001, 23 de janeiro de 1986. Disponível em: <http://www.mma.gov.br/port/conama/res/res86 /res0186.html>. Acesso em: 20 abr. 2017.

Brasil. Instrução Normativa IBAMA $n^{\circ} 146$, de 11 de janeiro de 2007. Disponível em: $<$ http://www.ibama.gov.br/licenciamento/modul os/arquivo.php?cod_arqweb=IN146>. Acesso em: 20 abr. 2017.

Campos, C. B.; Esteves, C. F.; Ferraz, K. M. P. M. B.; Crawshaw Jr., P. G.; Verdade, L. M. Diet of free ranging cats and dogs in a suburban and rural environment, Southeastern Brazil. Journal of Zoology, v. 273, n. 1, p. 14-20, 2007. https://dx.doi.org/10.1111/j.1469-7998.2007. 00291.x

CETESB - Companhia Ambiental do Estado de São Paulo. 2016. Disponível em: $<$ http://licenciamentoambiental.cetesb.sp.gov.br /eia-rima/>. Acesso em: 31 jan. 2016. 
Chiarello, A. G.; Aguiar, L. M. S.; Cerqueira, R.; Melo, F. R.; Rodrigues, F. H. G.; Silva, V. M. F. Mamíferos. In: Machado, A. B. M.; Drummond, G. M.; Paglia, A. P. (Orgs.). Livro Vermelho da Fauna Brasileira Ameaçada de Extinção. 1 ed. Brasília: MMA, 2008. v. 2.

Cullen Jr., L.; Bodmer, E. R.; Valladares-Padua, C. Ecological consequences of hunting in Atlantic forest patches, São Paulo, Brazil. Oryx, $\begin{array}{llll}\text { v. } 35, & \text { n. } 2, & \text { p. } 137-144, & 2001 .\end{array}$ https://dx.doi.org/10.1046/j.1365-3008.2001. 00163.x

Cullen Jr. L.; Rudran, R. Transectos lineares na estimativa de densidade de mamíferos e aves de médio e grande porte. In: Cullen Jr., L.; Rudran, R.; Valladares-Padua, C. (Orgs.). Métodos de estudo em biologia da conservação e manejo da vida silvestre. Curitiba: Editora UFPR, 2003.

Dale, V. H.; Beyeler, S. C. Challenges in the development and use of ecological indicators. Ecological Indicators, v. 1, n. 1, p. 3-10, 2001. https://dx.doi.org/10.1016/S1470-160X(01) 00003-6

Dias, E. G. C. S.; Sánchez, L. E. Deficiências na implementação de projetos submetidos à avaliação de impacto ambiental no Estado de São Paulo. Revista de Direito Ambiental, v. 6, n. 23, p. 163-204, 2001.

Galetti, M.; Sazima, I. Impacto de cães ferais em um fragmento urbano de Floresta Atlântica no sudeste do Brasil. Natureza \& Conservação, v. 4, n. 1, p. 58-63, 2006.

Gheler-Costa, C.; Verdade, L. M.; Almeida, A. F. Mamíferos não-voadores do campus "Luiz de Queiroz”, Universidade de São Paulo, Piracicaba, Brasil. Revista Brasileira de Zoologia, v. 19, Suppl. 2, p. 203-214, 2002. https://dx.doi.org/10.1590/S0101-817520020 00600021

Glasson, J.; Salvador, N. N. B. EIA in Brazil: a procedures-practice gap. A comparative study with reference to the European Union, and especially the UK. Environmental Impact Assessment Review, v. 20, n. 2, p. 191-225, $2000 . \quad$ https://dx.doi.org/10.1016/S01959255(99)00043-8

Hegel, C. G. Z.; Marini, M. A. Impact of the wild boar, Sus scrofa, on a fragment of Brazilian Atlantic Forest. Neotropical Biology and Conservation, v. 8, n. 1, p. 17-24. 2013. https://dx.doi.org/10.4013/nbc.2013.81.03

Khera, N.; Kumar, A. Inclusion of biodiversity in environmental impact assessments (EIA): a case study of selected EIA reports in India. Impact Assessment and Project Appraisal, $\begin{array}{lll}\text { v. 28, } & \text { n. 3, } 189-200, & \text { p. }\end{array}$ https://dx.doi.org/10.3152/146155110X1277298 2841005

Loss, S. R.; Will, T.; Marra, P. P. The impact of free-ranging domestic cats on wildlife of the United States. Nature Communications, v. 4, p. 1396, 2013. https://dx.doi.org/10.1038/ ncomms2380

Lyra-Jorge, M. C.; Ciocheti, G.; Pivello, V. R. Carnivore mammals in a fragmented landscape in Northeast of São Paulo State, Brazil. Biodiversity and Conservation, v. 17, n. 7, p. 1573-1580, 2008. https://dx.doi.org/10.1007/ s10531-008-9366-8

Lyra-Jorge, M. C.; Ribeiro, M. C.; Ciocheti, G.; Tambosi, L. R.; Pivello, V. R. Influence of multi-scale landscape structure on the occurrence of carnivorous mammals in a human-modified savanna, Brazil. European Journal of Wildlife Research, v. 56, p. 359368, 2010. https://dx.doi.org/10.1007/s10344009-0324-x

Lyra-Jorge, M. C.; Gheler-Costa, C.; Piña, C. I.; Rosalino, L. M.; Verdade, L. M. Wildlife surveys in agricultural landscapes: terrestrial medium to large-sized mammals. In: Verdade, L. M.; Lyra-Jorge, M. C.; Piña, C. I. (Eds.). Applied Ecology and Human Dimensions in Biological Conservation. London: Springer, 2014.

Magioli, M.; Moreira, M. Z.; Ferraz, K. M. P. M. B.; Miotto, R. A.; Camargo, P. B.; Rodrigues, M. G.; Canhoto, M. C. S.; Setz, E. F. Stable isotope evidence of Puma concolor (Felidae) feeding patterns in agricultural landscapes in Southeastern Brazil. Biotropica, v. 46, n. $4, \quad$ p. 451-460, 2014a. https://dx.doi.org/10.1111/btp.12115

Magioli, M.; Ferraz, K. M. P. M. B.; Rodrigues, M. G. Medium and large-sized mammals of an isolated Atlantic Forest remnant, Southeast São Paulo State, Brazil. Check List, v. 10, n. 4, p. 850-856, 2014b. Disponível em: $<$ https://www.biotaxa.org/cl/article/viewFile/10. 4.850/9735>. Acesso em: 7 ago. 2017.

Magioli, M.; Ferraz, K. M. P. M. B.; Setz, E. Z. F.; Percequillo, A. R.; Rondon, M. V. D. S. S.; Kuhnen, V. V.; Canhoto, M. C. S.; Santos, K. E. A.; Kanda, C. Z. K.; Fregonezi, G. L.; Prado, H. A.; Ferreira, M. K.; Ribeiro, M. C.; Villela, P. M. S.; Coutinho, L. L.; Rodrigues, M. G. Connectivity maintain mammal assemblages functional diversity within agricultural and fragmented landscapes. European Journal of Wildlife Research, v. 62, n. 4, p. 431-446, 
2016. https://dx.doi.org/10.1007/s10344-0161017-x

Magioli, M.; Ribeiro, M. C.; Ferraz, K. M. P. M. B.; Rodrigues, M. G. Thresholds in the relationship between functional diversity and patch size for mammals in the Brazilian Atlantic Forest. Animal Conservation, v. 18, n. 6, p. 499-511, 2015. https://dx.doi.org/10.1111/ acv.12201

Magurran, A. E. Measuring biological diversity. Oxford: Blackwell, 2004.

Marques, T. S.; Lara, N. R.; Camargo, P. B.; Verdade, L. M.; Martinelli, L. A. The use of stable isotopes analysis in wildlife studies. In: Verdade, L. M.; Lyra-Jorge, M. C.; Piña, C. I. (Eds.). Applied Ecology and Human Dimensions in Biological Conservation. London: Springer, 2014.

Martins, R. P.; Lewinsohn, T. M.; Diniz-Filho, J. A. F.; Coutinho, F. A.; da Fonseca, G. A. B. Drumond, M. A. Rumos para a formação de ecólogos no Brasil. Revista Brasileira de PósGraduação, v. 4, n. 7, p. 25-41, 2007. https://dx.doi.org/10.21713/2358-2332.2007.v4. 118

Mazzolli, M.; Jesus, E. D.; Wasem, R. W. S.; Borba, R. D.; Benedet, R.; Lessmann, K. G. Critical review of mammalogy studies in projects of power plants in the Plateau of Santa Catarina, Brazil. Natureza \& Conservação, v. 6, p. 199-209, 2008.

MMA - Ministério do Meio Ambiente. Download de dados geográficos disponíveis em: $<$ http://mapas.mma.gov.br/i3geo/datadownload. htm>. Acesso em: 10 mar. 2017.

Niemi, G. J.; McDonald, M. E. Application of ecological indicators. Annual Review of Ecology, Evolution, and Systematics, v. 35, p. 89-111, 2004. https://doi.org/10.1146/ annurev.ecolsys.35.112202.130132

Palmeira, F. B. L.; Trinca, C. T. Jaguar poisoning in southern Brazilian Amazonia. Cat News, v. 57, p. 9-11, 2012.

Pardini, R.; Ditt, E. H.; Cullen Jr. L.; Bassi, C.; Rudran, R. Levantamento rápido de mamíferos terrestres de médio e grande porte. In: Cullen Jr., L., Rudran, R., Valladares-Padua, C. (Orgs.). Métodos de estudo em biologia da conservação e manejo da vida silvestre. Curitiba: Editora UFPR, 2003.

Pardini, R.; Rocha, P. L. B.; El-Hani, C.; Pardini, F. Challenges and opportunities for bridging the research-implementation gap in ecological science and management in Brazil. In: Sodhi, N. S.; Gibson, L.; Raven, P. H.
(Orgs.). Conservation Biology: lessons from the tropics. Oxford, UK: Wiley-Blackwell, 2013. p. 77-85.

Paschoal, M.; Galetti, M. Seasonal food use by the neotropical squirrel Sciurus ingrami in Southeastern Brazil. Biotropica, v. 27, n. 2, p. 268-273, 1995. https://dx.doi.org/10.2307/ 2389006

Sánchez, L. E. Avaliação de impacto ambiental. São Paulo: Oficina de Textos, 2006.

Sánchez, L. E.; Croal, P. Environmental impact assessment, from Rio-92 to Rio+20 and beyond. Ambiente \& Sociedade, v. 15, n. 3, p. 41-54, $2012 . \quad$ https://dx.doi.org/10.1590/S1414753X2012000300004

Santos, A. J. Estimativa de riqueza de espécies. In: Cullen Jr., L.; Rudran, R.; Valladares-Padua, C. (Orgs.). Métodos de estudo em biologia da conservação e manejo da vida silvestre. Curitiba: Editora UFPR, 2003.

São Paulo. Decisão de Diretoria $n^{0}$ 217/2014/I, de 6 de agosto de 2014. Dispõe sobre a aprovação e divulgação do "Manual para Elaboração de Estudos para o Licenciamento Ambiental com Avaliação de Impacto Ambiental no âmbito da CETESB”. Disponível em: <http://www.cetesb.sp.gov.br/wp-content/ uploads/sites/11/2014/12/DD-217-14.pdf>.

Acesso em: 7 de ago 2017.

São Paulo. Decisão de Diretoria $\mathbf{n}^{\circ}$ 167/2015/C, de 13 de julho de 2015. Estabelece "Procedimento para a Elaboração dos Laudos de Fauna Silvestre para Fins de Licenciamento Ambiental e/ou Autorização para Supressão de Vegetação Nativa”, e dá outras providências. Disponível em: $<$ http://www.cetesb.sp.gov.br/wp-content/ uploads/sites/11/2014/12/DD-167-2015-C-semassinaturas.pdf> . Acesso em: 7 ago. 2017.

São Paulo. Lei $\mathbf{n}^{0}$ 13.542, de 8 de maio de 2009. Disponível em: <http://www.al.sp.gov.br/ repositorio/legislacao/lei/2009/lei-13542-

08.05.2009.html>. Acesso em: 7 ago. 2017.

São Paulo. Portaria DEPRN no 42, de 23 de outubro de 2000. Disponível em: $<$ http://licenciamento.cetesb.sp.gov.br/legislaca o/estadual/portarias/2000_Port_DEPRN_42.pdf >. Acesso em: 7 ago. 2017.

Scherer, M. Análise da qualidade técnica dos estudos de impacto ambiental em ambientes de Mata Atlântica de Santa Catarina: abordagem faunística. Biotemas, v. 24, n. 4, p. 171-181, 2011. https://dx.doi.org/10.5007/21757925.2011v24n4p171 
Schilling, A. C.; Batista, J. L. F.; Couto, H. T. Z. Ausência de estabilização da curva de acumulação de espécies em florestas tropicais. Ciência Florestal, v. 22, n. 1, p. 101-111, 2012. https://dx.doi.org/10.5902/198050985083

Silveira, L. F.; Beisiegel, B. M.; Curcio, F. F.; Valdujo, P. H.; Dixo, M.; Verdade, V. K.; Mattox, G. M. T.; Cunningham, P. T. M. What use do fauna inventories serve? Estudos Avançados, v. 24, n. 68, p. 173-207, 2010. https://dx.doi.org/10.1590/S0103-401420100 00100015

Srbek-Araujo, A. C.; Chiarello, A. G. Influence of camera-trap sampling design on mammal species capture rates and community structures in Southeastern Brazil. Biota Neotropica, v. 13, n. 2, p. 51-62, 2013. https://dx.doi.org/ 10.1590/S1676-06032013000200005

Straube, F. C.; Vasconcelos, M. F.; UrbenFilho, A.; Cândido-Jr, J. F. Protocolo mínimo para levantamentos de avifauna em Estudos de Impacto Ambiental. In: Matter, S. V.; Straube, F. C.; Accordi, I.; Piacentini, V.; Cândido-Jr, J. F. Ornitologia e conservação: ciência aplicada, técnicas de pesquisa e levantamento. Rio de Janeiro: Technical Books, 2010.

Teresa, B. T.; Casatti, L. Trait-based metrics as bioindicators: responses of stream fish assemblages to a gradient of environmental degradation. Ecological Indicators, v. 75, p. 249-258, 2017. https://dx.doi.org/10.1016/ j.ecolind.2016.12.041

Umetsu, F.; Metzger, J. P.; Pardini, R. Importance of estimating matrix quality for modeling species distribution in complex tropical landscapes: a test with Atlantic forest small mammals. Ecography, v. 31, n. 3, p. 359370, 2008. https://dx.doi.org/10.1111/j.09067590.2008.05302.x

Verdade, L. M.; Gheler-Costa, C.; Penteado, M.; Dotta, G. The impacts of sugarcane expansion on wildlife in the State of São Paulo, Brazil. Journal of Sustainable Bioenergy Systems, $\quad$ v. 2, p. 138-144, 2012. https://dx.doi.org/10.4236/jsbs.2012.24020

Vasconcelos, M. F. Uma opinião crítica sobre a qualidade e a utilidade dos trabalhos de consultoria ambiental sobre avifauna. Atualidades Ornitológicas, v. 131, p. 10-12, 2006.

Wegner, A.; Moore, S. A.; Bailey, J. Consideration of biodiversity in environmental impact assessment in Western Australia: practitioner perceptions. Environmental Impact Assessment Review, v. 25, n. 2, p. 143162, 2005. https://doi.org/10.1016/j.eiar.2004. 03.003

Informação da Licença: Este é um artigo Open Access distribuído sob os termos da Licença Creative Commons Attribution, que permite uso irrestrito, distribuição e reprodução em qualquer meio, desde que a obra original seja devidamente citada. 